\title{
Genotypic determinants of fluoroquinolone and macrolide resistance in Neisseria gonorrhoeae
}

\author{
Catherine L. Hall ${ }^{\mathrm{A}}$, Mark A. Harrison ${ }^{\mathrm{A}}$, Marcus J. Pond ${ }^{\mathrm{A}}$, Christine Chow $^{\mathrm{A}}$, \\ Emma M. Harding-Esch ${ }^{\mathrm{A}, \mathrm{B}}$ and S. Tariq Sadiq ${ }^{\mathrm{A}, \mathrm{B}, \mathrm{C}, \mathrm{D}}$

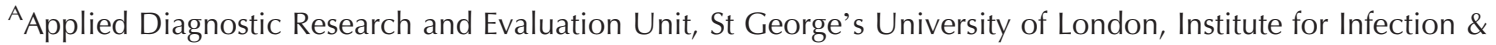 \\ Immunity, Cranmer Terrace, Tooting, London SW17 ORE, UK. \\ ${ }^{B}$ National Infection Service, Public Health England, 61 Colindale Avenue, London NW9 5EQ, UK. \\ CSt George's University Hospitals NHS Foundation Trust, Blackshaw Road, Tooting, London SW17 0QT, UK. \\ ${ }^{\mathrm{D}}$ Corresponding author. Email: ssadiq@sgul.ac.uk
}

\begin{abstract}
Background: High rates of antimicrobial resistance (AMR) in Neisseria gonorrhoeae hinder effective treatment, but molecular AMR diagnostics may help address the challenge. This study aimed to appraise the literature for resistance-associated genotypic markers linked to fluoroquinolones and macrolides, to identify and review their use in diagnostics. Methods: Medline and EMBASE databases were searched and data pooled to evaluate associations between genotype and phenotypic resistance. The minimum inhibitory concentration (MIC) cut-offs were $\leq 0.06 \mathrm{mg} \mathrm{L}^{-1}$ for non-resistance to ciprofloxacin and $\leq 0.5 \mathrm{mg} \mathrm{L}^{-1}$ for non-resistance to azithromycin. Results: Diagnostic accuracy estimates were limited by data availability and reporting. It was found that: 1) S91 and D95 mutations in the GyrA protein independently predicted ciprofloxacin resistance and, used together, gave $98.6 \%$ (95\% confidence interval (CI) 98.0-99.0\%) sensitivity and $91.4 \%(95 \%$ CI $88.6-93.7 \%)$ specificity; 2$)$ the number of $23 \mathrm{~S}$ rRNA gene alleles with C2611T or A2059G mutations was highly correlated with azithromycin resistance, with mutation in any allele giving a sensitivity and specificity of $66.1 \%(95 \% \mathrm{CI} 62.1-70.0 \%)$ and $98.9 \%(95 \% \mathrm{CI} 97.5-99.5 \%)$ respectively. Estimated negative (NPV) and positive predictive values (PPV) for a $23 \mathrm{~S}$ rRNA diagnostic were $98.6 \%(95 \% \mathrm{CI} 96.8-99.4 \%)$ and $71.5 \%$ (95\% CI 68.0-74.8\%) respectively; 3) mutation at amino acid position G45 in the MtrR protein independently predicted azithromycin resistance; however, when combined with 23S rRNA, did not improve the PPV or NPV. Conclusions: Viable candidates for markers of resistance detection for incorporation into diagnostics were demonstrated. Such tests may enhance antibiotic stewardship and treatment options.
\end{abstract}

Additional keywords: 23S rRNA, azithromycin, ciprofloxacin, gyrA, sexually transmissible infections.

Received 29 November 2018, accepted 1 April 2019, published online 1 August 2019

\section{Introduction}

Patients attending sexual health clinics (SHCs) will often present with non-specific genital infections, associated with several common symptoms, resulting from a diverse range of aetiologies. Effective infection management is therefore highly dependent on an accurate and rapid diagnosis to inform prompt treatment. However, this treatment is threatened by increasing antimicrobial resistance (AMR) rates within sexually transmitted pathogens, ${ }^{1}$ exemplified by recent UK reports of gonorrhoea resistant to azithromycin. ${ }^{2,3}$

Current diagnostics commonly use nucleic acid amplification tests (NAATs), favoured due to high sensitivity of detection and a quicker time to result than culture. ${ }^{4}$ However, culture remains the only means of determining AMR profiles for gonococcal infection, but its use is in decline and results are often unavailable at time of diagnosis, meaning empirical management of genital syndromes is common. ${ }^{5}$ Although empirical therapy helps to reduce time to treatment, onward transmission of infection and the possible loss to follow up, it also risks induction and further spread of AMR. Furthermore, it removes the opportunity for recycling of older antibiotics that are likely to still be effective in many cases, but are no longer recommended for empirical use. , $^{6,7}$

These challenges could, in part, be addressed by the development and deployment of NAAT technologies that identify both infection and AMR susceptibility in the laboratory and at the point-of-care (PoC), thereby enabling immediate administration of specific antibiotic therapy when patients are diagnosed ('precision medicine'). Calls for such novel diagnostics have been increasing in response to rising AMR rates ${ }^{8,9}$ yet have been restricted, in part, by incomplete understanding of the relationship between bacterial genotype and antimicrobial susceptibility phenotype.

Neisseria gonorrhoeae is a common sexually transmissible infection (STI), with high rates of AMR. ${ }^{10,11}$ Fluoroquinolone 
and macrolide antibiotics, which represent both current and previously recommended treatments for gonorrhoea, ${ }^{11}$ are effective in eradicating susceptible strains and are associated with specific genetic mechanisms of resistance. Macrolides inhibit protein synthesis by binding bacterial $23 \mathrm{~S}$ rRNA. Consequently, mutations associated with resistance typically centre on alteration of the macrolide binding site, through mutation or methylation of specific sites in the $23 \mathrm{~S}$ rRNA. ${ }^{12} N$. gonorrhoeae harbours four copies of the $23 \mathrm{~S}$ rRNA allele, and the number of alleles carrying mutations can rapidly increase under antibiotic selection pressure, assumed to be a result of internal homologous recombination. ${ }^{13,14}$ Fluoroquinolones inhibit DNA gyrase and DNA topoisomerase IV, two enzymes required for DNA replication, encoded by the gyrA and parC genes respectively. Specific mutations in the quinolone resistancedetermining regions (QRDR) of these genes are known to reduce the activity of fluoroquinolones. ${ }^{15,16}$ Mutations in genes encoding multi-drug efflux pumps that remove antibiotics from the bacteria can also influence minimum inhibitory concentrations (MIC) to varying degrees. ${ }^{17}$

As part of a body of work developing PoC tests for fluoroquinolone and macrolide resistance in Mycoplasma genitalium and $N$. gonorrhoeae (www.preciseresearch.co.uk), this review aimed to appraise the literature on AMR genotype for $N$. gonorrhoeae in relation to in vitro antimicrobial susceptibility to fluoroquinolone and macrolide antibiotics. These antibiotics were selected as they have relatively simple major mechanisms of resistance and a significant proportion of circulating gonococcal strains remain susceptible to them. ${ }^{11}$ For other antibiotics, such as cephalosporins, many different resistance mechanisms exist, affecting multiple genes with multiple mutations, ${ }^{13}$ making them more challenging for PoC AMR tests, where the current state of technology may limit screening to a finite number of mutations. We aimed to test the strength of genotypic-phenotypic associations when pooling data from included publications and to appraise the diagnostic accuracy of detecting AMR using the identified genotypic markers, in order to assess their suitability for inclusion on diagnostic platforms for AMR prediction.

\section{Methods}

\section{Publication search strategy and screening criteria}

Two separate searches were performed to reflect the aims: (1) macrolide resistance in $N$. gonorrhoeae; and (2) fluoroquinolone resistance in $N$. gonorrhoeae. Preliminary review of the literature informed search term format, including the organism name, region associated with resistance (e.g. 23S rRNA for macrolides or gyrA and parC for fluoroquinolones) and a broader component comprising variations on 'genotype' and the target antibiotic. This approach was used in an effort to ensure more general or emerging resistance mechanisms could still be detected (Text S1 available as Supplementary Material to this paper). Publication screening and data extraction were shared between C. L. Hall and M. A. Harrison and performed in September 2016 using OvidSP to search both EMBASE and Medline databases.

Abstracts were screened to determine publication suitability for inclusion in the review (Table 1), with the full text searched where necessary. Included publications had to target the organism of interest and antibiotic of interest, and report resistance-associated genotype in relation to antibiotic of interest, with no date restriction applied. Exclusion criteria were: publications that were not available in the English language or reviews and conference abstracts where results

Table 1. Number of publications included in the literature review and data analysis following screening of results from the literature search

\begin{tabular}{|c|c|c|c|}
\hline \multicolumn{2}{|l|}{ Inclusion/exclusion criteria } & \multirow{2}{*}{$\begin{array}{c}\text { Macrolide resistance } \\
\text { in Neisseria } \\
\text { gonorrhoeae }\end{array}$} & \multirow{2}{*}{$\begin{array}{c}\begin{array}{c}\text { Fluoroquinolone } \\
\text { resistance in } \\
\text { Neisseria } \\
\text { gonorrhoeae }\end{array} \\
613\end{array}$} \\
\hline & $\begin{array}{l}\text { Number of studies following the literature search, duplicate } \\
\text { deletion and reference and citation checking }\end{array}$ & & \\
\hline \multirow[t]{3}{*}{ Inclusion criteria not met } & Targets Neisseria gonorrhoeae & 52 & 49 \\
\hline & Discusses macrolide or fluoroquinolone resistance & 243 & 137 \\
\hline & $\begin{array}{l}\text { Reports resistance-associated genotype in relation to antibiotic } \\
\text { of interest }\end{array}$ & 153 & 239 \\
\hline \multirow[t]{4}{*}{ Exclusion criteria (literature review) } & Not available in English language & 21 & 24 \\
\hline & $\begin{array}{l}\text { Review data (bibliography and citation check still performed if } \\
\text { relevant) }\end{array}$ & 52 & 22 \\
\hline & $\begin{array}{l}\text { Conference abstracts where results are listed in a full } \\
\text { publication }\end{array}$ & 1 & 8 \\
\hline & Number of studies in literature review & 62 & 133 \\
\hline \multirow[t]{5}{*}{ Exclusion criteria (data analysis) } & $\begin{array}{l}\text { Studies listing mutations at gene level only (unless resistance is } \\
\text { the result of gene presence. e.g. } \mathrm{erm})^{\mathrm{A}}\end{array}$ & 3 & 8 \\
\hline & Non-clinical samples (e.g. laboratory and reference strains) ${ }^{\mathrm{A}}$ & 10 & 21 \\
\hline & Repeat datasets & 7 & 23 \\
\hline & Cannot determine number of isolates for each genotype & 0 & 3 \\
\hline & Number of studies in literature review used in data analysis & 42 & 79 \\
\hline
\end{tabular}

\footnotetext{
${ }^{\mathrm{A}}$ These included strains selected due to known mutation profiles (e.g. for testing of new methodology or antibiotics).
} 
were available in full publication. Due to variations in data provision and quality, further exclusion criteria were applied to limit publications to those providing the level of detail required for data analysis of phenotypic-genotypic relationships. Publications excluded at this stage were limited to the literature review only and used as a source of additional information for included papers, especially if two publications were linked. Exclusion criteria for data analysis were: mutation listed to gene level only; number of isolates or samples with each mutation not stated; reference strains and laboratory strains only; and repeat datasets. Some publications containing data constituting an exclusion criteria (e.g. reference strains) were extracted if this was clearly differentiated from the usable sample set. Publications included for data analysis (Text S2) and relevant reviews underwent reference and citation checking.

\section{Data extraction}

Optimal data capture from each eligible publication included: genotyping methodology; coverage and capacity to detect all mutations [e.g. targeted single nucleotide polymorphisms (SNPs) or sequencing of relevant gene regions]; whether samples were randomly selected or stratified by phenotypic characteristics; in vitro susceptibility phenotype; position of mutation; new base or amino acid following mutation; treatment regimen and dose; and whether the study had pre- and posttreatment data available. All samples or isolates were treated as separate cases, except repeat datasets and those identified as pre- and post- treatment samples (Table S3 and Table S4 available as Supplementary Material to this paper).

If the MIC to several fluoroquinolones or macrolides was reported, analysis centred on ciprofloxacin and azithromycin respectively, as these are either in use or have been recommended for treatment previously. Numbering of nucleotide bases or amino acid residue positions are in Escherichia coli numbering for $23 \mathrm{~S}$ rRNA resistance and $N$. gonorrhoeae numbering for fluoroquinolone resistance and mutations within the mtr locus.

\section{Data analyses}

Variation in the level of detail provided in each publication made comparison of the entire database unreliable, so set criteria were defined for each analysis (Table 2), with studies, samples or isolates only included if these were met.

In order to standardise the thresholds for resistance and susceptibility for isolates of $N$. gonorrhoeae, the European Committee on Antimicrobial Susceptibility Testing (EUCAST) guidelines ${ }^{18}$ were referenced to assign resistance phenotypes based on MICs described in each publication. EUCAST guidelines define ciprofloxacin resistance in $N$. gonorrhoeae as a $\mathrm{MIC}>0.06 \mathrm{mg} \mathrm{L}^{-1}$, which was used for this analysis. However, many studies used $\leq 0.06 \mathrm{mg} \mathrm{L}^{-1}$ as a susceptibility threshold; therefore, not differentiating between intermediate $\left(0.06 \mathrm{mg} \mathrm{L}^{-1}\right)$ and sensitive $\left(\leq 0.03 \mathrm{mg} \mathrm{L}^{-1}\right)$ isolates, as defined by EUCAST. Therefore, all isolates with an $\mathrm{MIC}$ of $\leq 0.06 \mathrm{mg} \mathrm{L}^{-1}$ were assigned as non-resistant. A similar approach was taken for azithromycin resistance in $N$. gonorrhoeae, with a MIC of $>0.5 \mathrm{mg} \mathrm{L}^{-1}$ assigned as resistant and $\leq 0.5 \mathrm{mg} \mathrm{L}^{-1}$ as non-resistant, with resistant isolates further categorised as low-level resistant $\left(>0.5 \mathrm{mg} \mathrm{L}^{-1}\right.$ and $\left.<2 \mathrm{mg} \mathrm{L}^{-1}\right)$, moderate-level resistant $\left(\geq 2 \mathrm{mg} \mathrm{L}^{-1}\right.$ and $\left.<256 \mathrm{mg} \mathrm{L}^{-1}\right)$ and high-level resistant $\left(\geq 256 \mathrm{mg} \mathrm{L}^{-1}\right)$.

\section{Statistical methods}

All statistical analysis was performed in Stata/IC 14 (Stata Corp, College Station, TX, USA). Both $\chi^{2}$ and univariate logistic regression analyses were performed for macrolide and fluoroquinolone resistance in $N$. gonorrhoeae to determine if presence of a mutation was significantly higher in isolates or samples with resistant MICs. This was followed by multivariate logistic regression analyses using univariate variables at $P<0.05$ in a forward stepwise approach to determine the strength of each genotype as independent markers of resistance. We chose a $0.05 P$-value threshold to limit the number of variables from chance associations being selected and considered the biological plausibility of the variables included (and not included) based on this cut-off. The correlation between the numbers of mutated 23S rRNA alleles for $N$. gonorrhoeae and level of macrolide resistance was analysed using Spearman's rank-order correlation coefficient. Sensitivity and specificity of resistance detection for the genotypic markers of AMR determined in this review were calculated using the following definitions: presence of an AMR marker in resistant isolates as true positive, in non-resistant isolates as false positive, their absence (wild-type) in nonresistant isolates as true negative and in resistant isolates as false negative. Calculated sensitivities and specificities were applied to the number of $N$. gonorrhoeae infections and the prevalence of resistance for 2016 in England and Wales acquired from Public Health England (PHE) STI and Gonococcal Resistance to Antimicrobial Surveillance Program (GRASP) datasets, ${ }^{11,19}$ to determine positive predictive values (PPV) and negative predictive values (NPV). Wilson score interval was used to calculate $95 \%$ confidence intervals (CI).

\section{Results}

\section{Macrolide resistance}

In $N$. gonorrhoeae, mutations within domain $\mathrm{V}$ of the $23 \mathrm{~S}$ rRNA gene, $\mathrm{A}-\mathrm{G}$ or $\mathrm{C}-\mathrm{T}$ substitution at A2059 and C2611 respectively, are associated with azithromycin resistance $(P<0.001)$. Of the 366 isolates harbouring 23S rRNA mutations with the specific number of mutated alleles reported, five were non-resistant and each had only one allele mutated. All isolates with two or more mutated alleles from this review were resistant $(n=359)$ (Table 3$)$.

We found data for 1015 isolates for which the numbers of 23S rRNA mutated alleles were recorded and for which an azithromycin resistance category (i.e. non-resistant, low-level, moderate-level and high-level resistant) could be allocated (Figure 1). A strong correlation was found between MIC and number of mutated alleles, in isolates where MIC was defined as an integer (e.g. not as a range) and the number of mutated alleles was specifically reported $\left(n=571, \mathrm{r}_{\mathrm{s}}=0.7846 ; P=<0.001\right)$.

A mutation in L22 was only reported once and mutations in L4 were not significantly associated with resistance. ${ }^{20}$ 


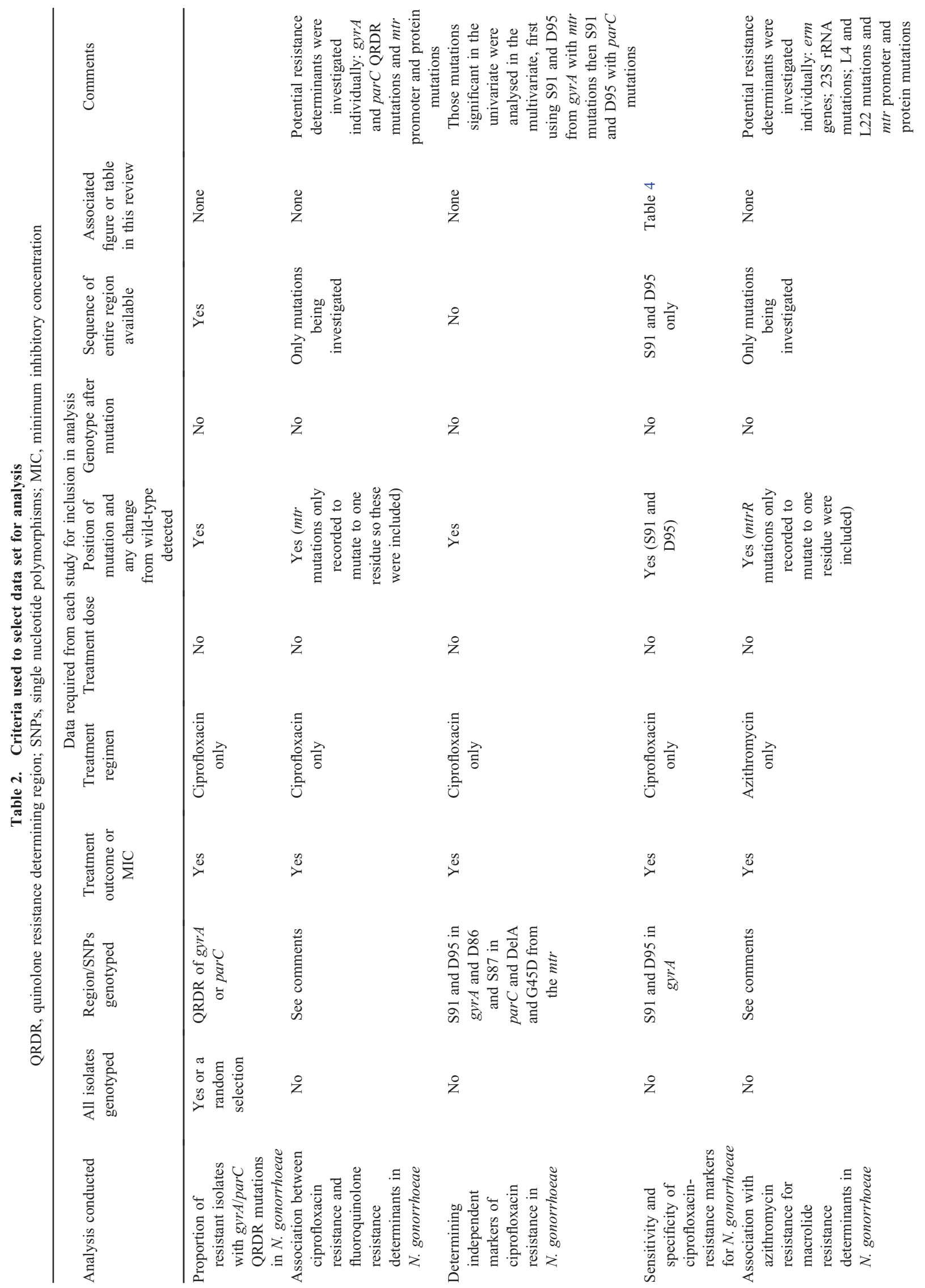




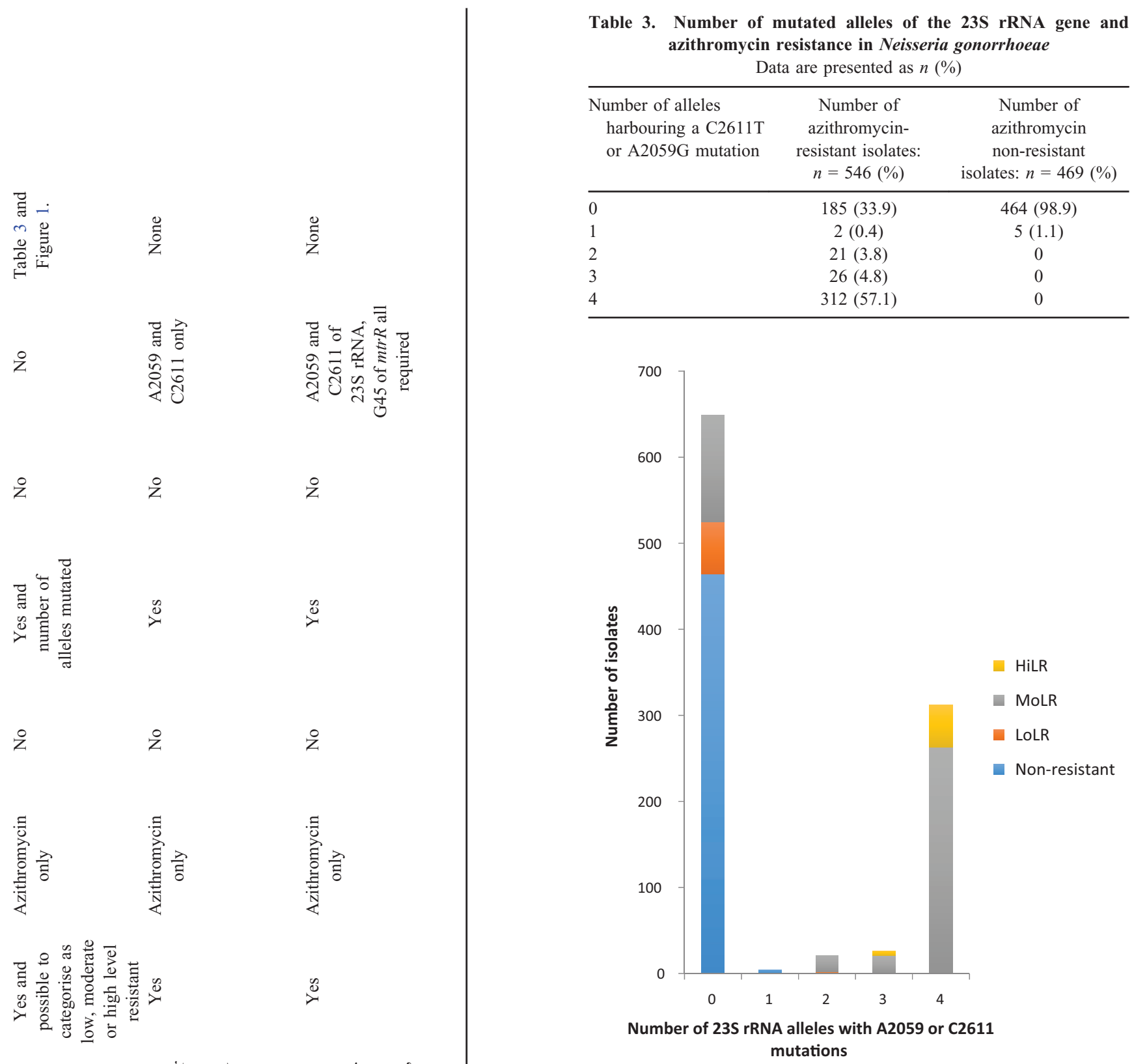

Fig. 1. Level of azithromycin resistance with number of mutated 23S rRNA alleles in Neisseria gonorrhoeae. Only isolates that could be categorised into macrolide resistance levels are included in the figure ( $n=1015$ ). Non-resistant: MIC $\leq 0.5 \mathrm{mg} \mathrm{L}^{-1}$; LoLR, low-level resistance: MIC $>0.5 \mathrm{mg} \mathrm{L}^{-1}$ and $<2 \mathrm{mg} \mathrm{L}^{-1}$; MoLR, moderate-level resistance: MIC $\geq 2 \mathrm{mg} \mathrm{L}^{-1}$ and $<256 \mathrm{mg} \mathrm{L}^{-1}$; HiLR, high-level resistance: $\mathrm{MIC} \geq 256 \mathrm{mg} \mathrm{L}^{-1}$. Spearman rank correlation coefficient $\left(\mathrm{r}_{\mathrm{s}}\right)$ between actual MIC and number of mutated alleles = 0.7846; $P=0.0000005(n=571)$. MIC, minimum inhibitory concentration.

Methylase (erm) genes, the mac efflux pump and ere genes were investigated, but were either not associated with resistance, very rare in resistant isolates or present with 23S rRNA mutations or where no other resistance associated region was typed.

Mutations in the MtrCDE transporter were found in both the repressor protein, MtrR, and its promoter. Mutations were 
identified at 20 positions within the MtrR, but only mutation at G45 was associated with resistance $(P<0.05)$. Alterations to amino acid position 105 in MtrR were omitted from the analysis, as the reference strains to which the isolates under analysis were being compared differed in the amino acid at this position, between histidine and tyrosine. This resulted in some studies considering H105 as wild-type whereas others considered Y105 as wild-type (Text S2). The 23S rRNA and G45 mutations could not be assessed together in multivariate logistic regression analysis, as in the sample set qualifying for this analysis; G45 mutations alone were no longer significantly associated with resistance. Furthermore, mutations at G45 were only present in $17.9 \%(145 / 812)$ of all resistant isolates screened.

Included publications described several rare alterations to the $m$ tr promoter region including: a Neisseria meningitidis-like promoter; mosaic promoter; and a range of insertions, deletions and substitutions. Most frequently reported alterations were an adenine deletion (DelA), a thymidine insertion and an adenineto-cytosine substitution within the mtr promoter region, but these changes were found in several both resistant and nonresistant isolates, and none were determined to be independent markers of resistance by univariate analysis.

Consequently, this review only indicated the A2059G and C2611T mutations within the 23S rRNA gene to be independent markers of azithromycin resistance, with higher levels of resistance more likely with increasing numbers of mutated alleles. However, the independent role of MtrR mutations on resistance could not be discounted. As a diagnostic marker of azithromycin resistance, use of the presence of either C2611T or A2059G 23S rRNA mutations $(n=1015)$ within at least one allele gave a sensitivity and specificity of $66.1 \%(95 \% \mathrm{CI}$ $62.1-70.0 \%)$ and $98.9 \% \quad(95 \%$ CI $97.5-99.5 \%)$ respectively; when mutations in two or more alleles were used, this gave $65.8 \%(95 \%$ CI $61.7-69.6 \%)$ and $100 \%$ $(95 \%$ CI $99.2-100 \%)$ respectively. When presence of either at least one mutated 23S rRNA allele or the MtrR mutation at G45 was considered, in a smaller sample set of 613 , sensitivity was $60.9 \%(95 \%$ CI $56.0-65.7 \%)$ and specificity $89.6 \%(95 \%$ CI $85.6-93.6 \%)$. The sensitivity and specificity of the combined putative diagnostic was applied to the $36244^{19}$ diagnoses of gonorrhoea made in England and Wales in 2016, using a prevalence of macrolide resistance (MIC $>0.5$ $\left.\mathrm{mg} \mathrm{L}^{-1}\right)$ of $10 \%$. ${ }^{11}$ This gave a PPV of $39.5 \%(95 \% \mathrm{CI}$ $38.2-40.8 \%)$ and a NPV of $95.4 \%$ (95\% CI $95.1-95.6 \%)$. However, when applying the lower margin of the $95 \%$ CI of sensitivity and specificity estimates, a PPV and NPV of $30.2 \%$ (95\% CI 29.1 - 31.3\%) and 94.6\% (95\% CI 94.3-94.9\%) were obtained respectively. Interestingly, applying the lower margins of accuracy of at least one $23 \mathrm{~S}$ rRNA mutant allele to this same dataset gave a PPV and NPV of $73.6 \%$ (95\% CI $72.0-75.2 \%)$ and $95.9 \%$ (95\% CI 95.6 - 96.1\%) respectively.

\section{Fluoroquinolone resistance}

Studies investigating fluoroquinolone resistance sequenced the QRDR for gyrA and parC, encoding the major subunits of DNA gyrase and DNA topoisomerase IV respectively. ${ }^{15}$ The minor subunits of these proteins, encoded by gyrB and parE respectively, were investigated in some cases and mutations detected. No mutations were found in $g y r B$, and in parE mutations were only present in isolates also harbouring gyr $A$ mutations.

Neisseria gonorrhoeae isolates frequently harboured multiple mutations in both genes, with a total of seven and nine amino acid changes within the QRDR of gyrA and parC respectively. Of these, mutations at S91 and D95 from gyr $A$ and D86 and S87 from parC were significantly higher in resistant isolates $(P<0.05)$, with wild-type S88 and E91 always found in non-resistant isolates. Mutations at other residues were present in less than $1 \%$ of the total number of resistant isolates where the QRDR was typed. Importantly, only one resistant isolate harboured a parC mutation $(\mathrm{E} 91 \mathrm{G})$ without an S91 and or D95 mutation in gyrA. Multivariate analyses revealed only S91 and D95 to be significantly associated with resistance, with $98.5 \%$ of resistant isolates harbouring one or both of these mutations (Table 4). Of those isolates with an S91 and D95 genotype and accompanying phenotypic susceptibility, $2.5 \%$ of resistant and $5.6 \%$ of non-resistant isolates harboured a D95 mutation only. Within this same sample set but using only those isolates where S91 is wild-type, D95 mutations were found in $64 \%$ of resistant isolates as opposed to $5.6 \%$ of non-resistant isolates. When used together, diversion from the wild-type at S91 or D95 gave a 98.6\% (95\% CI 98.0 - 99.0\%) sensitivity and 91.4\% (95\% CI 88.6 - 93.7\%) specificity for resistance detection.

The GRASP report for 2016 uses a MIC of $\geq 1.0 \mathrm{mg} \mathrm{L}^{-1}$ as the definition for ciprofloxacin resistance. ${ }^{11}$ As the EUCAST MIC of $>0.06 \mathrm{mg} \mathrm{L}^{-1}$ definition was used in this review, the $29 \%$ prevalence of resistance from GRASP could not be applied to the 36244 gonococcal diagnoses reported in England and Wales in 2016. ${ }^{19}$ However, speculating a resistance prevalence of $50 \%$ by our definition is not unreasonable, and applying the lower 95\% CI margins of sensitivity and specificity to the dataset gives a PPV and NPV of $89.5 \% \quad(95 \%$ CI $89.1-90.0 \%)$ and $97.7 \%$ (95\% CI $97.5-98.0 \%)$ respectively.

As with macrolide resistance, the MtrCDE efflux pump was investigated for association with ciprofloxacin resistance in $N$. gonorrhoeae, although in fewer studies. Of the 10 mutations reported within MtrR, only the G45D polymorphism was significantly higher in resistant isolates $(P<0.001)$, as was the DelA $m t r$ promoter mutation $(P<0.001)$. When S91, G45 and DelA were assessed together in a multivariate analysis, only mutations at S91 remained significantly associated with resistance.

Table 4. Mutations within DNA gyrase at codons S91 and D95 and association with fluoroquinolone resistance in Neisseria gonorrhoeae Data are presented as $n(\%)$

\begin{tabular}{lcc}
\hline & $\begin{array}{c}\text { gyrA } \mathrm{S} 91 \text { and D95 genotype and ciprofloxacin } \\
\text { susceptibility }(n=2678)\end{array}$ \\
Genotype & $\begin{array}{c}\text { Number of resistant } \\
\text { isolates }\end{array}$ & $\begin{array}{c}\text { Number of non-resistant } \\
\text { isolates } \\
(\%): n=2211\end{array}$ \\
\hline S91 and D95 mutation & $1702(77.0)$ & $6(1.3)$ \\
S91 mutation only & $421(19.0)$ & $8(1.7)$ \\
D95 mutation only & $56(2.5)$ & $26(5.6)$ \\
Wild-type at both residues & $32(1.5)$ & $427(91.4)$
\end{tabular}




\section{Discussion}

The spread of antimicrobial resistance threatens to undermine management of many infectious diseases. Interventions proposed to address this challenge include a more intelligent use of antibiotics, partly enabled by novel diagnostic technologies that can predict AMR rapidly. ${ }^{1}$ These promise more accurate treatments and the potential to recycle older antibiotics, thus improving antibiotic stewardship. ${ }^{21}$ In this review, we sought to appraise the literature for genotypic candidates associated with macrolide and fluoroquinolone resistance to $N$. gonorrhoeae and assess the potential accuracy of these candidate markers if included in AMR diagnostic platforms.

In $N$. gonorrhoeae, fluoroquinolones have been largely discontinued for empirical therapy because of high levels of resistance globally, yet in many regions a large proportion of gonococcal strains remain phenotypically susceptible to ciprofloxacin. ${ }^{11}$ Our analysis confirmed the central role of two mutations in the gyrA gene, representing amino acid changes at S91 and D95, which were both independently predictive of ciprofloxacin resistance. The data in this review gave a combined S91 and D95 diagnostic a sensitivity and specificity of $98.6 \%(95 \%$ CI $98.0-99.0 \%)$ and $91.4 \%(95 \%$ CI 88.6 - 93.7\%) respectively, for detection of ciprofloxacin resistance. In order to get a sense of the effect of use of these tests in clinical practice, we applied the lower 95\% CI margins of sensitivity and specificity to the 36244 gonococcal diagnoses reported in England and Wales in 2016. These gave a PPV and NPV of $89.5 \%$ (95\% CI $89.1-90.0 \%$ ) and $97.7 \%$ (95\% CI 97.5 - 98.0\%) respectively. This would imply a $97.7 \%$ chance of having a ciprofloxacin-susceptible infection if these markers were wild-type. In this virtual scenario, of the 18122 ciprofloxacin 'non-resistant' diagnoses of gonorrhoea, the S91 and D95 diagnostic test would potentially identify 16047 . The test would also report approximately 370 diagnoses to be genotypically 'non-resistant' when, in fact, phenotypically resistant, making such patients at risk of being given ciprofloxacin when they have a ciprofloxacin-resistant infection. This is an important clinical risk to minimise, and perhaps a related question to resolve would be at what NPV would it be better to give ciprofloxacin in combination with another antibiotic as opposed to risk monotherapy. Furthermore, among the 2016 cohort, 2075 genotypically resistant infections would, in fact, be phenotypically nonresistant. This second error represents both missed opportunities to identify patients for whom ciprofloxacin could be used and diagnostic test wastage. Clearly, these predictions are dependent on the availability of an AMR genotypic result at the point of diagnosis before treatment, population prevalence of resistance, as well as the molecular accuracy of marker detection. Evaluating these diagnostic approaches prospectively for accuracy, cost-effectiveness and effect on resistance spread will be an important factor in future development, in order to minimise the risks identified above. However, the data suggest that an S91 and D95 gyrA diagnostic may well be of value, even in particularly high ciprofloxacin resistance prevalence settings, but more work on the determinants of resistance is required to improve both sensitivity and specificity.
Although critical for resistance in $E$. coli, the D95 mutation was reported in borderline ciprofloxacin-resistant $N$. gonorrhoeae in transformation studies or when induced in vitro, but the MIC was 16-fold greater than the wild-type parental strain. $^{22,23}$ Of those isolates wild-type at S91 and resistant in this review, 64\% carried a mutation at D95, compared with just $5.6 \%$ in non-resistant strains, with inclusion of D95 raising sensitivity of detecting AMR from $96.0 \%(95 \%$ CI $95.1-96.8 \%$ ) to $98.5 \%$ (95\% CI $98.0-99.0 \%)$. Inclusion of D95 reduces specificity from $97.1 \%$ (95\% CI $95.2-$ $98.3 \%$ ) to $91.4 \%$ (95\% CI 88.6 - 93.7\%), meaning more isolates will be labelled resistant when in fact non-resistant, but as ciprofloxacin is no longer recommended for treatment of $N$. gonorrhoeae, these people would not have received this treatment anyway, highlighting this as an added value approach.

In this review, we demonstrated a clear association between 23S rRNA mutations at positions A2059 and C2611 and azithromycin resistance in $N$. gonorrhoeae, particularly if two or more alleles of the mutated gene were carried. Although the sensitivity of using 23S rRNA markers for detecting azithromycin resistance was only $66.1 \%$, this could be explained by the association of mutated alleles with moderate or high-level resistance, combined with the fact that a significant proportion of resistant samples were low-level resistant (Figure 1). Increased azithromycin MICs in relation to increasing numbers of mutated $23 \mathrm{~S}$ rRNA alleles has been reported previously in $N$. gonorrhoeae and other species harbouring multiple copies of the 23S rRNA gene. ${ }^{14,24}$ Each gene copy encodes for a $23 \mathrm{~S}$ rRNA protein, with the likely mechanism of phenotypic resistance occurring when a single mutated 23S rRNA gene drives the same mutations in the other copies through a process of internal recombination, reducing the proportion of antibiotic susceptible 23S rRNA protein available. $^{14}$

Applying the lower margin of the $95 \%$ CI of sensitivity and specificity estimates to the $36244^{19}$ diagnoses of gonorrhoea made in England and Wales in 2016 gave a PPV and NPV of $30.2 \%$ and $94.6 \%$ respectively, for a combined $23 \mathrm{~S}-\mathrm{G} 45$ test. Interestingly, applying the lower margins of accuracy of at least one 23S rRNA mutant allele to this same dataset gave a PPV and NPV of $73.6 \%$ and $95.9 \%$ respectively. For clinicians, the estimates suggest that for azithromycin resistance, some value may be obtained by using the $23 \mathrm{~S}$ rRNA markers alone to identify azithromycin susceptible cases genotypically, but that this would come at a significant cost in terms of test wastage as a result of misidentifying susceptible cases as resistant. As with ciprofloxacin, how azithromycin might be used with a $23 \mathrm{~S}$ rRNA only test could be explored, perhaps, for example, using it in combination therapies and at high doses (Figure 1) following a negative test, as our findings demonstrate that high-level resistance is effectively ruled out. However, such an approach should give caution because of the number of moderate-level resistant infections in this group. Again, further work to better understand the genotypic-phenotypic relationships of macrolide AMR may improve the accuracy of predictions. The lack of consensus between the different reference strains used by studies, in terms of the amino acid at position 105 within MtrR, prevented further analysis of this mutation. Uniform agreement on the wild-type and mutant 
genotype at this position would allow further analysis and support better understanding of this residue in relation to azithromycin resistance.

Overexpression of MtrCDE can increase MIC to macrolides ${ }^{13,17}$ and has been associated with fluoroquinolone resistance when in combination with gyrA or $\operatorname{parC}$ mutations. ${ }^{25,26}$ However, this review found mutations within $\mathrm{MtrCDE}$ and its promoter unreliable as targeted independent markers of azithromycin and ciprofloxacin resistance due to their prevalence in non-resistant isolates or association with more definitive resistance markers such as S91. This demonstrates the complicated nature of designing AMR detection systems when several mechanisms can influence the resistance phenotype, and a limitation of using specific targets for resistance detection. Alternative methods such as sequencing may be able to screen several regions associated with resistance and use a wild-type genotype across the entire region as a marker of susceptibility. Furthermore, variation in MIC reporting necessitated resistance cut-offs, which may mean smaller borderline increases or intermediate MICs associated with certain resistance mechanisms, are missed.

Rapid NAAT diagnostics have been integrated into SHC clinical care pathways, ${ }^{27}$ but in the UK, there remains no licenced diagnostic for dual detection of infection and susceptibility at the $\mathrm{PoC}$, although these are under investigation; for example, the Precise study (www. preciseresearch.co.uk) and the SpeeDx assay (https://plexpcr. com/resistanceplus-gc/). Furthermore, use and trials of laboratory detection of fluoroquinolone resistance in $N$. gonorrhoeae are underway. ${ }^{28}$ However, implementation and technology choice for the detection of resistance markers presents a series of challenges. SNP-based resistance detection may require coverage of multiple alleles, such as the $23 \mathrm{~S}$ rRNA region in $N$. gonorrhoeae, ${ }^{13}$ or account for the occurrence of synonymous SNPs to avoid false calling of resistant isolates. Test efficacy is also highly dependent on active surveillance programs, monitoring patterns of resistance and associated genotypes, ideally on a global scale to capture the influence of regional differences in antibiotic usage.

This review provides a critical appraisal of genetic determinants of resistance to fluoroquinolones and macrolides in $N$. gonorrhoeae; however, data reporting varied widely between publications, with differences in both resistance mechanisms and specific mutations tested. Furthermore, as MIC cut-offs used for classification of phenotypic resistance vary globally, we selected resistance cut-offs based on EUCAST guidelines and developed clear criteria for data inclusion in each analysis. However, data collected from these studies are still subject to common limitations, including sensitivity and specificity of molecular tests, particularly for studies investigating new methods for resistance detection, although the majority of publications used sequencing or established PCR-based assays. In consideration of this, we used the lower CIs of sensitivities and specificities to calculate conservative PPV and NPV estimates.

We were also limited to using in vitro MIC as the measure of resistance to the antibiotic of interest, as this is the most common means of reporting antibiotic susceptibility in $N$. gonorrhoeae. As in vitro AMR does not necessarily equate to treatment failure, increased reporting of treatment response data alongside in vitro susceptibility testing would be important to future work assessing the utility of these markers for clinical impact.

Molecular detection of antibiotic resistance offers the potential to significantly enhance NAAT diagnostics. Providing susceptibility profiles at the PoC enables a more prompt and accurate treatment, an invaluable tool in aiding antibiotic stewardship and a key approach to reduction of AMR. However, such tests should be used to enhance testing and be performed in conjunction with culture and sequencing to monitor susceptibility profiles and circulating and emerging genotypes.

\section{Conflicts of interest}

Ms Hall, Dr Pond and Ms Chow disclose having received funding outside the submitted work from: Atlas Genetics, Alere, Cepheid and Sekisui. Mr Harrison, Dr Harding-Esch and Dr Sadiq disclose having received funding outside the submitted work from: Atlas Genetics, Alere, Cepheid, SpeedDx, Mologic and Sekisui. Dr Harding-Esch discloses their membership of the Becton- Dickinson 'Provision of Sexual Health in the UK' Advisory Board.

\section{Acknowledgements}

This work was supported by the UK Clinical Research Collaboration (Medical Research Council) (http://www.ukcrc.org/) Translation Infection Research Initiative Consortium (grant number G0901608) and by the National Institute for Health Research (NIHR) i4iProgram (https://www.nihr.ac.uk/about-us/how-we-aremanaged/boards-and-panels/ program-boards436and-panels/invention-for-innovation/) (grant number II-LB-0214-20005). The views expressed are those of the authors and not necessarily those of the NIHR, the NHS or the Department of Health. Both grants were awarded to S. Tariq Sadiq.

\section{References}

1 The Review on Antimicrobial Resistance. Tackling drug-resistant infections globally: final report and recommendations. London: Review on Antimicrobial Resistance; 2016. Available online at: http://amr-review.org/sites/default/files/160518_Fina1\%20paper_ with\%20cover.pdf [verified 3 November 2015].

2 Chisholm SA, Wilson J, Alexander S, Tripodo F, Al-Shahib A, Schaefer U, Lythgow K, Fifer H. An outbreak of high-level azithromycin resistant Neisseria gonorrhoeae in England. Sex Transm Infect 2016; 92(5): 365-7. doi:10.1136/sextrans-2015052312

3 Eyre DW, Sanderson ND, Lord E, Regisford-Reimmer N, Chau K, Barker L, Morgan M, Newnham R, Golparian D, Unemo M, Crook DW, Peto TE, Hughes G, Cole MJ, Fifer H, Edwards A, Andersson MI. Gonorrhoea treatment failure caused by a Neisseria gonorrhoeae strain with combined ceftriaxone and high-level azithromycin resistance, England, February 2018. Euro Surveill 2018; 23(27): 1800323. doi:10.2807/1560-7917.ES.2018.23.27.1800323

4 Low N, Unemo M. Molecular tests for the detection of antimicrobial resistant Neisseria gonorrhoeae: when, where, and how to use? Curr Opin Infect Dis 2016; 29(1): 45-51. doi:10.1097/QCO.000000 0000000230

5 Mohammed H, Ison CA, Obi C, Chisholm S, Cole M, Quaye N, Hughes G; GRASP Collective Group. Frequency and correlates of culture-positive infection with Neisseria gonorrhoeae in England: a 
review of sentinel surveillance data. Sex Transm Infect 2015; 91(4): 287-93. doi:10.1136/sextrans-2014-051756

6 Manhart LE, Gillespie CW, Lowens MS, Khosropour CM, Colombara DV, Golden MR, Hakhu NR, Thomas KK, Hughes JP, Jensen NL, Totten PA. Standard treatment regimens for nongonococcal urethritis have similar but declining cure rates: a randomized controlled trial. Clin Infect Dis 2013; 56(7): 934-42. doi:10.1093/cid/cis1022

7 Sadiq ST, Dave J, Butcher PD. Point-of-care antibiotic susceptibility testing for gonorrhoea: improving therapeutic options and sparing the use of cephalosporins. Sex Transm Infect 2010; 86(6): 445-6. doi:10.1136/sti.2010.044230

8 Gaydos C, Hardick J. Point of care diagnostics for sexually transmitted infections: perspectives and advances. Expert Rev Anti Infect Ther 2014; 12(6): 657-72. doi:10.1586/14787210.2014.880651

9 Unemo M.. Challenges with antimicrobial susceptibility testing for Neisseria gonorrhoeae in the era of extensively drug-resistant gonorrhoea - molecular antimicrobial resistance testing crucial. Pathog Glob Health 2014; 108(5): 214-5. doi:10.1179/20477724 14Z.000000000216

10 Ohnishi M, Golparian D, Shimuta K, Saika T, Hoshina S, Iwasaku K, Nakayama S, Kitawaki J, Unemo M. Is Neisseria gonorrhoeae initiating a future era of untreatable gonorrhea?: detailed characterization of the first strain with high-level resistance to ceftriaxone. Antimicrob Agents Chemother 2011; 55(7): 3538-45. doi:10.1128/AAC.00325-11

11 Public Health England. GRASP 2016 Report. Surveillance of antimicrobial resistance in Neisseria gonorrhoeae. London: Public Health England; 2016.

12 Leclercq R. Mechanisms of resistance to macrolides and lincosamides: nature of the resistance elements and their clinical implications. Clin Infect Dis 2002; 34(4): 482-92.

13 Unemo M, Shafer WM. Antimicrobial resistance in Neisseria gonorrhoeae in the 21st century: past, evolution, and future. Clin Microbiol Rev 2014; 27(3): 587-613. doi:10.1128/CMR.00010-14

14 Chisholm SA, Dave J, Ison CA. High-level azithromycin resistance occurs in Neisseria gonorrhoeae as a result of a single point mutation in the 23S rRNA genes. Antimicrob Agents Chemother 2010; 54(9): 3812-6. doi:10.1128/AAC.00309-10

15 Belland RJ, Morrison SG, Ison C, Huang WM. Neisseria gonorrhoeae acquires mutations in analogous regions of gyrA and parC in fluoroquinolone-resistant isolates. Mol Microbiol 1994; 14 (2): 371-80. doi:10.1111/j.1365-2958.1994.tb01297.x

16 Redgrave LS, Sutton SB, Webber MA, Piddock LJ. Fluoroquinolone resistance: mechanisms, impact on bacteria, and role in evolutionary success. Trends Microbiol 2014; 22(8): 438-45. doi:10.1016/ j.tim.2014.04.007

17 Chitsaz M, Brown MH. The role played by drug efflux pumps in bacterial multidrug resistance. Essays Biochem 2017; 61(1): 127-39. doi:10.1042/EBC20160064
18 The European Committee on Antimicrobial Susceptibility Testing. Breakpoint tables for interpretation of MICs and zone diameters. Version 7.1. Basel: European Society of Clinical Microbiology and Infectious Diseases; 2017. Available online at: http://www.eucast.org [verified 3 November 2015].

19 Public Health England. Sexually transmitted infections and chlamydia screening in England 2016. London: Public Health England; 2017.

20 Zaman S, Fitzpatrick M, Lindahl L, Zengel J. Novel mutations in ribosomal proteins L4 and L22 that confer erythromycin resistance in Escherichia coli. Mol Microbiol 2007; 66(4): 1039-50. doi:10.1111/ j.1365-2958.2007.05975.x

21 Bissonnette L, Bergeron MG. Infectious disease management through point-of-care personalized medicine molecular diagnostic technologies. J Pers Med 2012; 2(2): 50-70. doi:10.3390/ jpm2020050

22 Tanaka M, Sakuma S, Takahashi K, Nagahuzi T, Saika T, Kobayashi I, Kumazawa J. Analysis of quinolone resistance mechanisms in Neisseria gonorrhoeae isolates in vitro. Sex Transm Infect 1998; 74 (1): 59-62. doi:10.1136/sti.74.1.59

23 Heisig P. Genetic evidence for a role of parC mutations in development of high-level fluoroquinolone resistance in Escherichia coli. Antimicrob Agents Chemother 1996; 40(4): 879-85. doi:10.1128/ AAC.40.4.879

24 Tait-Kamradt A, Davies T, Cronan M, Jacobs MR, Appelbaum PC, Sutcliffe J. Mutations in 23S rRNA and ribosomal protein L4 account for resistance in pneumococcal strains selected in vitro by macrolide passage. Antimicrob Agents Chemother 2000; 44(8): 2118-25. doi:10.1128/AAC.44.8.2118-2125.2000

25 Ilina EN, Vereshchagin VA, Borovskaya AD, Malakhova MV, Sidorenko SV, Al-Khafaji NC, Kubanova AA, Govarun VM. Relation between genetic markers of drug resistance and susceptibility profile of clinical Neisseria gonorrhoeae strains. Antimicrob Agents Chemother 2008; 52(6): 2175-82. doi:10.1128/ AAC.01420-07

26 Balashov S, Mordechai E, Adelson ME, Gygax SE. Multiplex bead suspension array for screening Neisseria gonorrhoeae antibiotic resistance genetic determinants in noncultured clinical samples. J Mol Diagn 2013; 15(1): 116-29.

27 Gaydos CA. Review of use of a new rapid real-time PCR, the Cepheid GeneXpert(R) (Xpert) CT/NG assay, for Chlamydia trachomatis and Neisseria gonorrhoeae: results for patients while in a clinical setting. Expert Rev Mol Diagn 2014; 14(2): 135-7. doi:10.1586/14737159. 2014.871495

28 Allan-Blitz LT, Humphries RM, Hemarajata P, Bhatti A, Pandori MW, Siedner MJ, Klausner JD. Implementation of a rapid genotypic assay to promote targeted ciprofloxacin therapy of Neisseria gonorrhoeae in a large health system. Clin Infect Dis 2017; 64(9): 1268-70. 